\title{
A note on symmetries of diffusions within a martingale problem approach
}

\author{
Francesco C. De Vecchi*, Paola Morando ${ }^{\dagger}$ and Stefania Ugolini ${ }^{\ddagger}$
}

\begin{abstract}
A geometric reformulation of the martingale problem associated with a set of diffusion processes is proposed. This formulation, based on second order geometry and Itô integration on manifolds, allows us to give a natural and effective definition of Lie symmetries for diffusion processes.
\end{abstract}

\section{Introduction}

The theory of infinitesimal symmetries of ordinary and partial differential equations (ODEs and PDEs respectively) is a classical research topic in applied mathematics, providing powerful tools both for investigating the qualitative behaviour of differential equations and for obtaining some explicit expression for their solutions (see, e.g., 17, 21]). The theory of symmetries of stochastic differential equations (SDEs) is, in comparison, less developed. There are two main approaches to this problem in the case of Brownian-motion-driven SDEs. The first approach, based on the Markovian property of solutions to a SDE, looks for the classical Lie symmetries of the Markov generator, which is an analytical object (see 1, 9, 15). The second method, directly inspired by Lie ideas, consists in seeking for some semimartingale transformations leaving invariant the set of solutions to the considered SDE (see, e.g. [6, 8, 12, 14, 20, 23]). Both approaches have their own strong and weak points: for example, the first method permits to treat a larger class of transformations and processes, while the second one results more convenient in order to generalize the deterministic notions of reduction and reconstruction by quadratures (see [2, 13]).

In [3] we propose a partial reconciliation of these two programs: in fact, despite working in the second method perspective, we introduce a larger class of SDEs transformations which permits both to include all the transformations of the first approach and to obtain all the applications of the second one.

In this article we make a synthesis of the above two approaches from a new prospective. In particular, starting from the martingale problem characterization of the solutions to a SDE, typical of the Markovian setting, we introduce, in the stochastic framework, a geometric formulation of the symmetry problem.

The main idea is to generalize the well known identification of an ODE on a manifold $M$ with a one-dimensional module $K$ on the tangent bundle of the zero-jet space $N=J^{0}(\mathbb{R}, M)=\mathbb{R} \times M$ (or, equivalently, a module $K^{\prime}$ of codimension one on the cotangent bundle $T^{*} N$ ). Thanks to this correspondence, the symmetries of an ODE can be identified as the family of diffeomorphisms $\bar{\Phi}: N \rightarrow N$ transforming the module $K$ (or, equivalently, the module $K^{\prime}$ ) into itself.

\footnotetext{
*Dip. di Matematica, Università degli Studi di Milano, via Saldini 50, Milano, email: francesco.devecchi@unimi.it

†DISAA, Università degli Studi di Milano, via Celoria 2, Milano, email: paola.morando@unimi.it

‡Dip. di Matematica, Università degli Studi di Milano, via Saldini 50, Milano, email: stefania.ugolini@unimi.it
} 
In order to generalize the previous (deterministic) geometric approach to the stochastic framework we need two main ingredients (both introduced by P.A. Meyer and L. Schwartz [16, 19] and thereafter studied by Emery [5]): second order geometry and Itô integration on manifolds. In particular, second order geometry allows us to introduce diffusors (a generalization of vector fields) and codiffusors (a generalization of differential forms), while Itô integration on manifolds permits to integrate any codiffusor $\lambda$ along a semimartingale $X$ defined on $M$.

In this framework the usual martingale problem associated with a second order operator $L$ can be reformulated in the following way: a semimartingale $X$ is a solution to the martingale problem associated with $L$ if and only if, for any $\lambda \in \Lambda_{L}$, the integral of $\lambda$ with respect to $X$ is a local martingale, where $\Lambda_{L}$ is the module of codiffusors annihilating $L$ (see Section 44). In this way we reinterpret the martingale problem in terms of a natural geometric object: the module of codiffusors $\Lambda_{L}$.

Therefore, we prove that a diffeomorphism $\bar{\Phi}$ is a symmetry for the martingale problem associated with $L$, which means that $\bar{\Phi}$ transforms solutions to the martingale problem into other solutions to the same martingale problem, if and only if the natural action of the pull-back $\bar{\Phi}^{*}$ transforms $\Lambda_{L}$ into itself. The last condition is purely geometrical and permits to explicitly compute the set of symmetries of the martingale problem associated with $L$ (see Section 5).

The paper is organized as follows: in Section 2 we briefly introduce second order geometry and Itô integration on manifolds and in Section 3 we study the behaviour of the geometric and probabilistic objects introduced in the previous section with respect to spatial diffeomorphisms and deterministic time changes. With this background, in Section 4, we propose a geometric reformulation of the martingale problem for diffusion processes and in Section [5 we exploit it in order to provide a suitable notion of symmetry and to explicitly compute the corresponding determining equations. Finally we compare the class of Lie symmetries arising from our approach with other ones appearing in the literature.

\section{Preliminaries: second order geometry and Itô integration on manifold}

In this section, also in order to fix notations, we briefly recall some basic facts about second order geometry and Itô integration on manifolds. The interested reader is referred to [5, 16, 19] for proofs and further details.

\subsection{Second order geometry}

Given a smooth manifold $M$, we denote by $C^{\infty}(M)$ the set of real-valued smooth functions defined on $M$. If $F$ is a bundle with base manifold $B$, we denote by $S(F)$ the set of smooth sections of $F$. Finally, if $M^{\prime}$ is a manifold and $n \in \mathbb{N}$, we denote by $J^{n}\left(M, M^{\prime}\right)$ the bundle of $n$ jets of $n$ times differentiable functions defined on $M$ and taking values in $M^{\prime}$.

Let $M$ be a smooth manifold and $u$ be a global coordinate defined on $\mathbb{R}$. The subset $u^{-1}(0) \subset$ $J^{2}(M, \mathbb{R})$ is a submanifold of $J^{2}(M, \mathbb{R})$ and actually a vector subbundle of $J^{2}(M, \mathbb{R})$.

Definition 2.1 The submanifold $u^{-1}(0) \subset J^{2}(M, \mathbb{R})$ is called the bundle of codiffusors of the manifold $M$ and is denoted by $\tau^{*} M$.

Given a coordinate system $x^{i}$ on $M$, let $\left(x^{i}, u, u_{x^{i}}, u_{x^{i} x^{j}}\right)$ be the associated coordinate system on $J^{2}(M, \mathbb{R})$. Denoting by $\pi^{2}: J^{2}(M, \mathbb{R}) \rightarrow M$ the projection of $J^{2}(M, \mathbb{R})$ into $M$, we define the smooth function $\Pi: J^{2}(M, \mathbb{R}) \rightarrow \tau^{*} M$ as

$$
\Pi\left(x^{i}, u, u_{x^{i}}, u_{x^{i} x^{j}}\right)=\left(x^{i}, 0, u_{x^{i}}, u_{x^{i} x^{j}}\right) .
$$


The projection $\left.\pi^{2}\right|_{\tau^{*} M}$ makes the submanifold $\tau^{*} M$ a vector subbundle of $J^{2}(M, \mathbb{R})$ with base $M$. The function $\Pi$ is well defined and is a morphism of vector bundles on $M$.

From now on we call codiffusor on $M$ a smooth section of the vector bundle $\tau^{*} M$.

Given a smooth function $f \in C^{\infty}(M)$, let $D^{2} f$ denote the natural lift of $f$ to $J^{2}(M, \mathbb{R})$ given in coordinates by

$$
D^{2} f(x)=\left(x^{i}, f(x), \partial_{x^{i}} f(x), \partial_{x^{i} x^{j}} f(x)\right) .
$$

Definition 2.2 We call second differential of $f \in C^{\infty}(M)$ the codiffusor

$$
d^{2} f:=\Pi\left(D^{2} f\right) .
$$

Moreover, for $f, g \in C^{\infty}(M)$, we denote by $d f \cdot d g$ the codiffusor

$$
d f \cdot d g:=\frac{1}{2}\left(d^{2}(f g)-g d^{2} f-f d^{2} g\right) .
$$

Since, from the previous definition, we have

$$
\begin{aligned}
d^{2} x^{i} & =\left(x^{i}, 0, u_{x^{j}}=\delta_{j}^{i}, u_{x^{i} x^{j}}=0\right) \\
d x^{i} \cdot d x^{j} & =\left(x^{i}, 0, u_{x^{l}}=0, u_{x^{k} x^{l}}=\frac{1}{2}\left(\delta_{k i} \delta_{l j}+\delta_{k j} \delta_{l i}\right)\right),
\end{aligned}
$$

we can give an explicit coordinate expression for $d^{2} f$ :

$$
d^{2} f=\partial_{x^{i}} f d^{2} x^{i}+\partial_{x^{i} x^{j}} f d x^{i} \cdot d x^{j} .
$$

Remark 2.3 If $\lambda$ is a codiffusor on $M$ and $x^{i}$ is a coordinate system on $M$, there exist unique functions $\lambda_{i}, \lambda_{i j}=\lambda_{j i} \in C^{\infty}(M)$ such that locally

$$
\lambda=\lambda_{i} d^{2} x^{i}+\lambda_{i j} d x^{i} \cdot d x^{j} .
$$

The following theorem provides a useful characterization of codiffusors on $M$.

Theorem 2.4 For any codiffusor $\lambda$ on $M$ there exist $g_{i}, f_{i} \in C^{\infty}(M), i=1, \ldots, m$, such that $\lambda=\sum_{i=1}^{m} g_{i} d^{2} f_{i}$.

Using Theorem 2.4 it is possible to extend the product between the differentials of functions given in Definition 2.2 to a product defined for any couple of differential one forms. In a coordinate system $x^{i}$ the product has the following representation

$$
\omega \cdot \gamma=\omega_{i} \gamma_{j} d x^{i} \cdot d x^{j}
$$

where $\omega=\omega_{i} d x^{i}$ and $\gamma=\gamma_{j} d x^{j}$.

Definition 2.5 We denote by $\tau M$ the dual bundle of $\tau^{*} M$ on $M$, and we call it the bundle of diffusors. A section of $\tau M$ is called a diffusor.

Given a system of coordinates $x^{i}$ on $M$, Remark 2.3 ensures that $\left\{d^{2} x^{i}, d x^{i} \cdot d x^{j}\right\}$ form a local basis of the fibers of $\tau^{*} M$. Hence, it is possible to introduce the local dual basis $\left\{\partial_{x^{i}}, \partial_{x^{i} x^{j}}\right\}$ so that

$$
\begin{aligned}
\left\langle d^{2} x^{i}, \partial_{x^{j}}\right\rangle & =\delta_{j}^{i} \\
\left\langle d x^{i} \cdot d x^{j}, \partial_{x^{k}}\right\rangle & =0 \\
\left\langle d^{2} x^{i}, \partial_{x^{j} x^{k}}\right\rangle & =0 \\
\left\langle d x^{i} \cdot d x^{j}, \partial_{x^{k} x^{m}}\right\rangle & =\frac{1}{2}\left(\delta_{k}^{i} \delta_{m}^{j}+\delta_{m}^{i} \delta_{k}^{j}\right)
\end{aligned}
$$


We remark that the use of symbols $\partial_{x^{i}}$ and $\partial_{x^{i} x^{j}}$ for the basis of $\tau M$ is not misleading, since the diffusors $\partial_{x^{i}}$ and $\partial_{x^{i} x^{j}}$ are closely related to the partial derivatives. Given a diffusor $L$ defined on $M$, it is natural to associate with $L$ the differential operator $L: C^{\infty}(M) \rightarrow C^{\infty}(M)$ defined by

$$
L(f):=\left\langle d^{2} f, L\right\rangle .
$$

The following result provides a characterization of diffusors through their associated differential operators.

Theorem 2.6 Given a diffusor $L$ on $M$, its associated operator $L: C^{\infty}(M) \rightarrow C^{\infty}(M)$ is a second order linear differential operator without multiplicative term. Conversely, if $\Lambda: C^{\infty}(M) \rightarrow C^{\infty}(M)$ is a second order linear differential operator without multiplicative term, there exists a unique diffusor $L$ on $M$ such that, $\forall f \in C^{\infty}(M)$,

$$
\Lambda(f)=L(f) .
$$

Given two vector fields $X, Y$ on $M$, we consider the second order operator $L_{X Y}$ defined by

$$
L_{X Y}(f)=X(Y(f)) .
$$

By Theorem 2.6 there exists a diffusor $L_{X Y} \in \tau M$ such that, if $X=X^{i} \partial_{x^{i}}$ and $Y=Y^{i} \partial_{x^{i}}$,

$$
L_{X Y}=X^{i} Y^{j} \partial_{x^{i} x^{j}}+X^{i}\left(\partial_{x^{i}} Y^{j}\right) \partial_{x^{j}}
$$

and we have

$$
L_{X Y}-L_{Y X}=[X, Y]
$$

\subsection{Itô integration on manifolds}

Given a filtered probability space $\left(\Omega, \mathcal{F},\left(\mathcal{F}_{t}\right)_{t \in[0, T]}, \mathbb{P}\right)$, in the following we consider only stochastic processes (processes for short) adapted with respect to the filtration $\mathcal{F}_{t}$. Moreover, all (local) martingales are always $\mathcal{F}_{t}$ (local) martingales.

Given a process $X$ and a stopping time $\tau$, we denote by $X^{\tau}$ the process stopped at $\tau$. Moreover, if $X$ and $Z$ are two real continuous semimartingales, their quadratic covariation is denoted by $[X, Z]$ (although this notation is the same as the above commutator of vectors fields, the different meaning will be clear from the contest).

Definition 2.7 An almost surely continuous process $X$ taking values in $M$ is a semimartingale if, $\forall f \in C^{\infty}(M), f(X)$ is a real continuous semimartingale.

Semimartingales represent the largest class of processes for which Itô integration can be introduced.

Theorem 2.8 Given a semimartingale $X$ on $M$, there exists a unique linear functional from $S\left(\tau^{*} M\right)$ into the space of real semimartingales, denoted by

$$
\lambda \longmapsto \int\left\langle\lambda, d X_{s}\right\rangle:=\int\left\langle\lambda\left(X_{s}\right), d X_{s}\right\rangle,
$$

such that, for $f \in C^{\infty}(M)$ and $\lambda \in S\left(\tau^{*} M\right)$,

- $\int\left\langle d^{2} f\left(X_{t}\right), d X_{t}\right\rangle=f(X)-f\left(X_{0}\right)$;

- $\int\left\langle f\left(X_{t}\right) \lambda\left(X_{t}\right), d X_{t}\right\rangle=\int f\left(X_{t}\right) d\left(\int\left\langle\lambda\left(X_{s}\right), d X_{s}\right\rangle\right)$, where the latter integral is the Itô integral along the real semimartingale $\int\left\langle\lambda\left(X_{s}\right), d X_{s}\right\rangle$. 
Remark 2.9 In Theorem 2.8 we define a stochastic integral satisfying

$$
\left(\int\left\langle\lambda, d X_{t}\right\rangle\right)_{0}=0
$$

However, it is easy to extend above definition so that $\left(\int\left\langle\lambda, d X_{t}\right\rangle\right)_{s}=0$ for some $s \in \mathbb{R}$.

Later on we adopt the notation

$$
\int_{s}^{t}\left\langle\lambda\left(X_{r}\right), d X_{r}\right\rangle=\left(\int\left\langle\lambda\left(X_{r}\right), d X_{r}\right\rangle\right)_{t}-\left(\int\left\langle\lambda\left(X_{r}\right), d X_{r}\right\rangle\right)_{s} .
$$

Some useful properties of the Itô integral are collected in the following proposition.

Proposition 2.10 Let $X$ be a semimartingale on $M, f, g \in C^{\infty}(M), \lambda, \sigma \in S\left(\tau^{*} M\right)$ and let $\tau$ be a stopping time. Then

- $\left(\int\left\langle\lambda\left(X_{t}\right), d X_{t}\right\rangle\right)^{\tau}=\int\left\langle\lambda\left(X_{t}^{\tau}\right), d X_{t}^{\tau}\right\rangle ;$

- $\int\left\langle(d f \cdot d g)\left(X_{t}\right), d X_{t}\right\rangle=\frac{1}{2}[f(X), g(X)]$.

\section{Transformations of codiffusors and Itô integrals}

In this section, in order to introduce a suitable notion of symmetry, we study the behaviour of codiffusors, diffusors and Itô integration under spatial transformations and deterministic time changes of the process $X$.

Let us fix some preliminary notations: given a smooth manifold $M$, we denote by $N=J^{0}(\mathbb{R}, M)=$ $\mathbb{R} \times M$ and we consider the time $t$ as the first coordinate of $N$.

\subsection{Transformations of diffusors and codiffusors}

The definition of codiffusors as sections of a suitable subbundle of $J^{2}(M, \mathbb{R})$ suggests the possibility of generalizing in a natural way the pull-back of smooth functions and differential forms to diffusors and codiffusors. The construction is purely geometric and is based on the following theorem.

Theorem 3.1 Given two smooth manifolds $M$ and $M^{\prime}$ and a smooth map $\Phi: M \rightarrow M^{\prime}$, there exists a unique map $\Phi^{*}: S\left(\tau^{*} M^{\prime}\right) \rightarrow S\left(\tau^{*} M\right)$ such that $, \forall f, g \in C^{\infty}\left(M^{\prime}\right)$ and $\forall \lambda, \sigma \in S\left(\tau^{*} M^{\prime}\right)$,

i) $\Phi^{*}\left(d^{2} f\right)=d^{2}\left(\Phi^{*}(f)\right)$,

ii) $\Phi^{*}(f \lambda+g \sigma)=\Phi^{*}(f) \Phi^{*}(\lambda)+\Phi^{*}(g) \Phi^{*}(\sigma)$.

Proof. The uniqueness follows from Theorem 2.4

To prove the existence, we set an atlas $\left\{U_{j}\right\}_{j \in \mathbb{N}}$ on $M^{\prime}$, with local coordinates $\left\{y_{j}^{i}\right\}$ in $U_{j}$ on $M^{\prime}$, and a partition of the unity $\left\{\phi_{j}\right\}$ subordinated to $\left\{U_{j}\right\}$. If the support $K$ of $\lambda \in S\left(\tau^{*} M^{\prime}\right)$ is contained in the support of $\phi_{j}$ and if

$$
\lambda=\lambda_{i} d^{2} y_{j}^{i}+\lambda_{i k} d y_{j}^{i} \cdot d y_{j}^{k}
$$

we define

$$
\Phi^{*}(\lambda)=\Phi^{*}\left(\lambda_{i}\right) d^{2} \Phi_{j}^{i}(x)+\Phi^{*}\left(\lambda_{i k}\right) d \Phi_{j}^{i}(x) \cdot d \Phi_{j}^{k}(x),
$$


where $\Phi_{j}^{i}(x)=\left(y_{j}^{i} \circ \Phi\right)(x)$. Note that, if $P \in M$ is not in $\Phi^{-1}(K)$, then $\Phi^{*}(\lambda)(P)=0$. If $\lambda$ is any codiffusor, then we define

$$
\Phi^{*}(\lambda):=\sum_{j} \Phi^{*}\left(\phi_{j} \lambda\right)
$$

The above relation is well defined as $\sum_{j} \Phi^{*}\left(\phi_{j} \lambda\right)$ is pointwise a finite sum. Moreover, it is easy to verify that $\Phi^{*}$ satisfies properties $i$ ) and $\left.i i\right)$.

Definition 3.2 Given a diffeomorphism $\Phi: M \rightarrow M^{\prime}$, the map $\Phi^{*}: S\left(\tau^{*} M^{\prime}\right) \rightarrow S\left(\tau^{*} M\right)$ is called the pull-back of codiffusors. The map $\Phi_{*}:\left(\tau^{*} M\right) \rightarrow S\left(\tau^{*} M^{\prime}\right)$ defined as $\Phi_{*}:=\left(\Phi^{-1}\right)^{*}$ is called the push-forward of codiffusors.

Theorem 3.3 Given a diffeomorphism $\Phi: M \rightarrow M^{\prime}$, there exists a unique map $\Phi^{*}: S\left(\tau M^{\prime}\right) \rightarrow$ $S(\tau M)$ such that, $\forall L \in S\left(\tau M^{\prime}\right)$ and $\forall \lambda \in S\left(\tau^{*} M\right)$,

$$
\left\langle\lambda, \Phi^{*}(L)\right\rangle=\Phi^{*}\left(\left\langle\Phi_{*}(\lambda), L\right\rangle\right) .
$$

Proof. Given $L \in S\left(\tau M^{\prime}\right)$, we consider the second order differential operator $L^{\prime}$ on $C^{\infty}(M)$ such that $\forall f \in C^{\infty}(M)$

$$
\left.L^{\prime}(f)=\Phi^{*} L\left(\Phi_{*}(f)\right)\right) .
$$

By Theorem 2.6 there exists a unique diffusor $L^{\prime} \in S(\tau M)$ such that

$$
L^{\prime}(f)=\left\langle d^{2} f, L^{\prime}\right\rangle .
$$

Then, by Theorem 2.4, we have $L^{\prime}=\Phi^{*}(L)$.

Definition 3.4 Given a diffeomorphism $\Phi: M \rightarrow M^{\prime}$, we call $\Phi^{*}: S\left(\tau M^{\prime}\right) \rightarrow S(\tau M)$ the pullback of diffusors and $\Phi_{*}: S(\tau M) \rightarrow S\left(\tau M^{\prime}\right)$ the push-forward of diffusors.

Remark 3.5 If $\Phi: M \rightarrow M^{\prime}$ is a smooth function, $\forall \mu, \sigma \in S\left(T^{*} M^{\prime}\right)$ we have

$$
\Phi^{*}(\mu \cdot \sigma)=\Phi^{*}(\mu) \cdot \Phi^{*}(\sigma) .
$$

Moreover, if $\Phi$ is invertible, $\forall X, Y \in S\left(T M^{\prime}\right)$

$$
\Phi^{*}\left(L_{X Y}\right)=L_{\Phi^{*}(X) \Phi^{*}(Y)}
$$

All the previous expressions hold when we replace $\Phi^{*}$ with $\Phi_{*}$.

If we consider a one-parameter group of diffeomorphisms $\Phi_{a}$ describing the flow of a vector field $X$, we can give the following definition.

Definition 3.6 Given a vector field $X$ on $M$, with corresponding one-parameter flow $\Phi_{a}$, the Lie derivative of a codiffusor (diffusor) $\lambda$ along $X$ is

$$
\mathcal{L}_{X} \lambda=\left[\frac{d}{d a}\left(\Phi_{a}^{*} \lambda\right)\right]_{a=0} .
$$

The following theorem permits to compute the Lie derivatives of many important objects.

Theorem 3.7 Let $X, X_{1}, X_{2}$ be three vector fields on $M, L$ a diffusor on $M, \lambda$ a codiffusor on $M$, $f$ a smooth function on $M, \mu, \sigma$ two differential forms and $\Phi: M \rightarrow M^{\prime}$ a diffeomorphism from $M$ onto $M^{\prime}$. Then 

1. $\mathcal{L}_{X}(f \lambda)=\mathcal{L}_{X}(f) \lambda+f \mathcal{L}_{X}(\lambda)$,
2. $\mathcal{L}_{X}(f L)=\mathcal{L}_{X}(f) L+f \mathcal{L}_{X}(L)$,
3. $\mathcal{L}_{X}(\langle\lambda, L\rangle)=\left\langle\mathcal{L}_{X}(\lambda), L\right\rangle+\left\langle\lambda, \mathcal{L}_{X}(L)\right\rangle$,
4. $\mathcal{L}_{X}\left(d^{2} f\right)=d^{2}\left(\mathcal{L}_{X}(f)\right)=d^{2}(X(f))$,
5. $\mathcal{L}_{X}(L)(f)=X(L(f))-L(X(f))$,
6. $\mathcal{L}_{X}(\mu \cdot \sigma)=\mathcal{L}_{X}(\mu) \cdot \sigma+\mu \cdot \mathcal{L}_{X}(\sigma)$,
7. $\mathcal{L}_{X}\left(L_{X_{1} X_{2}}\right)=L_{\left[X, X_{1}\right] X_{2}}+L_{X_{1}\left[X, X_{2}\right]}$,
8. $\mathcal{L}_{\Phi_{*} X}\left(\Phi_{*} \lambda\right)=\Phi_{*}\left(\mathcal{L}_{X}(\lambda)\right)$,
9. $\mathcal{L}_{\Phi_{*} X}\left(\Phi_{*} L\right)=\Phi_{*}\left(\mathcal{L}_{X}(L)\right)$.

Proof. The proof is an easy application of the properties of the pull-back of diffusors and codiffusors and of the Leibniz rule for the derivative of a product.

From Theorem 3.7 we obtain the explicit coordinate expression of the Lie derivative of diffusors and codiffusors along a vector field $X$. In particular, if $X=\phi^{i} \partial_{x^{i}}, L=A^{i j} \partial_{x^{i} x^{j}}+b^{i} \partial_{x^{i}}$ and $\lambda=\lambda_{i} d^{2} x^{i}+\lambda_{i j} d x^{i} \cdot d x^{j}$, we have

$$
\begin{gathered}
\mathcal{L}_{X}(L)=\left(\phi^{k} \partial_{x^{k}} A^{i j}-A^{i k} \partial_{x^{k}} \phi^{j}-A^{k j} \partial_{x^{k}} \phi^{i}\right) \partial_{x^{i} x^{j}} \\
+\left(\phi^{k} \partial_{x^{k}} b^{i}-b^{k} \partial_{x^{k}} \phi^{i}-A^{j k} \partial_{x^{j} x^{k}} \phi^{i}\right) \partial_{x^{i}} . \\
\mathcal{L}_{X} \lambda=\left(\phi^{k} \partial_{x^{k}} \lambda_{i j}+\lambda_{i k} \partial_{x^{j}} \phi^{k}+\lambda_{k j} \partial_{x^{i}} \phi^{k}+\lambda_{k} \partial_{x^{i} x^{j}} \phi^{k}\right) d x^{i} \cdot d x^{j} \\
+\left(\phi^{k} \partial_{x^{k}} \lambda_{i}+\lambda_{k} \partial_{x^{i}} \phi^{k}\right) d^{2} x^{i} .
\end{gathered}
$$

In order to generalize the geometric approach to symmetry problem from ODEs to diffusion processes, it is useful to give the following definition.

Definition 3.8 A subset $\Gamma$ of $S(\tau M)$ (or $S\left(\tau^{*} M\right)$ ) is a module of dimension $k$ if

1. $\forall L_{1}, L_{2} \in \Gamma$ also $L_{1}+L_{2} \in \Gamma$,

2. $\forall L \in \Gamma$ and $f \in C^{\infty}(M)$ we have $f L \in \Gamma$,

3. for each point $P$ there exist a neighborhood $U$ of $P$ and $k$ diffusors (codiffusors) $L_{1}, \ldots, L_{k} \in \Gamma$ such that, $\forall L \in \Gamma$, we have $L=\sum_{i=1}^{k} f_{i} L_{i}$ in $U$, where $f_{1}, \ldots, f_{k}$ are suitable functions in $C^{\infty}(M)$. Furthermore, for any $Q \in U, L_{1}(Q), \ldots, L_{k}(Q)$ are $k$ linearly independent elements of $\tau_{Q} M\left(\right.$ or $\left.\tau_{Q}^{*} M\right)$.

In particular, given $L \in S(\tau M)$ such that $L(P) \neq 0$ for all $P \in M$, we can consider the one-dimensional module

$$
\mathfrak{L}_{L}=\left\{f L \mid f \in C^{\infty}(M)\right\}
$$

and its annihilator, i.e. the set of codiffusors

$$
\Lambda_{L}=\left\{\lambda \in S\left(\tau^{*} M\right) \mid\langle\lambda, L\rangle=0\right\}
$$

which is a module of rank $(m-1)$, where $m=\operatorname{rank}\left(\tau^{*} M\right)$. 
Definition 3.9 Let $\Gamma$ be a k-dimensional module on $M$. A diffeomorphism $\Phi: M \rightarrow M$ is a symmetry of $\Gamma$ if $\Phi_{*}(\Gamma)=\Gamma$. A complete vector field $X \in S(T M)$ is an infinitesimal symmetry of $\Gamma$ if the flow $\Phi_{a}$ of $X$ is a symmetry of $\Gamma$ for all $a \in \mathbb{R}$.

Theorem 3.10 A complete vector field $X$ is a symmetry of $\Gamma$ if and only if, $\forall L \in \Gamma$, we have $\mathcal{L}_{X} L \in \Gamma\left(\right.$ or simply $\left.\mathcal{L}_{X}(\Gamma) \subseteq \Gamma\right)$.

Proof. We give only a sketch of the proof; further details can be found in 3 .

If $\Phi_{a, *}(\Gamma)=\Gamma$, evaluating in zero the derivatives with respect to $a$, we get $\mathcal{L}_{X}(\Gamma) \subseteq \Gamma$.

Conversely, suppose that $\mathcal{L}_{X}(\Gamma) \subseteq \Gamma$. Let $L_{1}, \ldots, L_{r}$ be local generators for $\tau M$ and choose $L_{i}$ such that, for $i=1, \ldots, k$, they are also local generators for the module $\Gamma$. Given a diffusor $L$, there exist some functions $\alpha_{1}, \ldots, \alpha_{k}$ and $\beta_{1}, \ldots, \beta_{r-k}$ (depending on $a$ and $x$ ) such that $\Phi_{a, *}(L)=$ $\sum_{i=1}^{k} \alpha_{i} L_{i}+\sum_{i=1}^{r-k} \beta_{i} L_{i+k}$. Since $\mathcal{L}_{X}(\Gamma) \subseteq \Gamma$, the functions $\alpha_{i}, \beta_{i}$ satisfy the following system of first order PDEs

$$
\left(\begin{array}{c}
\partial_{a}(\alpha) \\
\partial_{a}(\beta)
\end{array}\right)=\left(\begin{array}{c}
X(\alpha) \\
X(\beta)
\end{array}\right)+\left(\begin{array}{cc}
A(x) & B(x) \\
0 & C(x)
\end{array}\right) \cdot\left(\begin{array}{c}
\alpha \\
\beta
\end{array}\right),
$$

where $\alpha=\left(\alpha_{1}, \ldots, \alpha_{k}\right), \beta=\left(\beta_{1}, \ldots, \beta_{r-k}\right)$ and $A(x), B(x), C(x)$ are suitable matrix-valued functions. Using the method of characteristics it is possible to prove that equation (9) admits a unique solution for any smooth initial value $\alpha(0), \beta(0)$. Moreover, the form of equation (9) ensures that, if $\beta(0)=0$, then $\beta(a)=0$ for any $a \in \mathbb{R}$. Hence, since $L \in \Gamma$, we have that $\beta(0)=0$ and $\Phi_{a, *}(L) \in \Gamma$ for any $a$.

\subsection{Itô integral and space and time transformations}

In the following we study the behaviour of a semimartingale under space and time transformations.

Proposition 3.11 Given two manifolds $M$ and $M^{\prime}$, a semimartingale $X$ on $M$ and a smooth function $\Phi: M \rightarrow M^{\prime}$, the process $X^{\prime}=\Phi(X)$, defined as $X_{t}^{\prime}=\Phi\left(X_{t}\right)$, is a semimartingale on $M^{\prime}$.

Proof. The proof is an easy consequence of the definition of semimartingale on a manifold.

In order to introduce time transformations, we consider a strictly increasing function $f \in$ $C^{\infty}(\mathbb{R})$, so that also $f^{-1}$ is a smooth strictly increasing function. If $X$ is a semimartingale on $M$, we denote by $X^{\prime}=H_{f}(X)$ the process

$$
X_{t^{\prime}}^{\prime}=H_{f}(X)_{t^{\prime}}:=X_{f^{-1}\left(t^{\prime}\right)}
$$

Moreover, working towards a unified description of space and time transformations, we consider a smooth map $\Phi: M \rightarrow M^{\prime}$, a deterministic time change $f$ and a semimartingale $X$ on $M$, and we define

$$
\Phi_{f}(X)=H_{f}(\Phi(X)) .
$$

Theorem 3.12 With the above notations, $\forall \lambda \in S\left(\tau^{*} M^{\prime}\right)$

$$
\int\left\langle\lambda\left(\Phi_{f}(X)_{t}\right), d \Phi_{f}(X)_{t}\right\rangle=H_{f}\left(\int\left\langle\Phi^{*}(\lambda)\left(X_{t}\right), d X_{t}\right\rangle\right) .
$$


Proof. We define the linear operator $I$ from $S\left(\tau^{*} M^{\prime}\right)$ into the set of real semimartingales such that

$$
I(\lambda)=H_{f}\left(\int\left\langle\Phi^{*}(\lambda)\left(X_{t}\right), d X_{t}\right\rangle\right) .
$$

Using Theorem 3.1. Theorem 2.8 and the definition of $\Phi_{f}$, we have

$$
\begin{aligned}
I\left(d^{2} g\right) & =H_{f}\left(\int\left\langle\Phi^{*}\left(d^{2} g\right)\left(X_{t}\right), d X_{t}\right\rangle\right) \\
& =H_{f}\left(\int\left\langle d^{2} \Phi^{*}(g)\left(X_{t}\right), d X_{t}\right\rangle\right) \\
& =H_{f}\left(g(\Phi(X))-g\left(\Phi(X)_{0}\right)\right)=g\left(\Phi_{f}(X)\right)-g\left(\Phi_{f}(X)_{0}\right),
\end{aligned}
$$

Furthermore, the change rule of Itô integral with respect to absolutely continuous time changes (see, e.g., 18, Proposition 30.10]) ensures that

$$
\begin{aligned}
I(g \lambda) & =H_{f}\left(\int\left\langle\Phi^{*}(g \lambda)\left(X_{t}\right), d X_{t}\right\rangle\right) \\
& =H_{f}\left(\int\left\langle\Phi^{*}(g)\left(X_{t}\right) \Phi^{*}(\lambda)\left(X_{t}\right), d X_{t}\right\rangle\right) \\
& =H_{f}\left(\int \Phi^{*}(g)\left(X_{t}\right) d\left(\int\left\langle\Phi^{*}(\lambda), d X_{s}\right\rangle\right)_{t}\right) \\
& =\int g\left(\Phi_{f}(X)_{t}\right) d I(\lambda)_{t} .
\end{aligned}
$$

Hence, using the characterization of Itô integral given in Theorem 2.8 , we have $I(\lambda)=\int\left\langle\lambda, d \Phi_{f}(X)\right\rangle$ and this completes the proof.

Given a semimartingale $X$ on $M$, the semimartingale

$$
\bar{X}_{t}=\left(t, X_{t}\right) \in N
$$

is called the lifting of $X$ to $N$. When $\lambda \in S\left(\tau^{*} N\right)$, we use the following notation

$$
\int\left\langle\lambda, d X_{t}\right\rangle:=\int\left\langle\lambda\left(\bar{X}_{t}\right), d \bar{X}_{t}\right\rangle
$$

Given a transformation $\bar{\Phi}: N \rightarrow N^{\prime}$, we write $\bar{\Phi}=(f, \Phi)$, where $f$ is the component of $\bar{\Phi}$ over $\mathbb{R}$ and $\Phi$ is the component of $\bar{\Phi}$ over $M$. If $f$ depends only on $t$ we say that $\bar{\Phi}=(f, \Phi)$ is projectable. We call semimartingale transformation any diffeomorphism $\bar{\Phi}$ which is projectable. We denote by $X^{\prime}=\Phi_{f}(\bar{X})$ the transformed semimartingale given by

$$
X_{t^{\prime}}^{\prime}=\Phi_{f}\left(f^{-1}\left(t^{\prime}\right), X_{f^{-1}\left(t^{\prime}\right)}\right) .
$$

Remark 3.13 The lifting $\bar{X}^{\prime}$ of $X^{\prime}$ to $N^{\prime}$ satisfies

$$
\overline{X^{\prime}}=H_{f}(\bar{\Phi}(\bar{X})) \text {. }
$$

Theorem 3.14 Let $\bar{\Phi}=(f, \Phi): N \rightarrow N^{\prime}$ be a semimartingale transformation, and $\lambda \in S\left(\tau^{*} N^{\prime}\right)$; then

$$
\int\left\langle\lambda, d \Phi_{f}(X)_{t}\right\rangle=H_{f}\left(\int\left\langle\bar{\Phi}^{*}(\lambda), d X_{t}\right\rangle\right) .
$$

Proof. The proof is a simple application of Theorem 3.12 and Remark 3.13 


\section{A novel formulation of the martingale problem via second order geometry}

It is well-known that the martingale problem approach, due to Stroock and Varadhan ([22]), represents a modern and fruitful way to introduce diffusion processes, alternative to the classical definition as SDEs solutions. For a complete exposition of the topic see [11, 22, (4, 10, for the manifolds setting). In the following we call $X$ a semimartingale starting at time $s \in \mathbb{R}$ if $X_{s+t}$ is a semimartingale (starting at time 0 ).

Definition 4.1 A semimartingale $D$ on $M$ starting at time $s$ is a solution to the martingale problem associated with a diffusor $L$ up to a stopping time $\tau>s$ if, $\forall g \in C^{\infty}(N)$, the real semimartingale $D^{g}$ given by

$$
D_{t}^{g}=g\left(\bar{D}_{t \wedge \tau}\right)-g\left(\bar{D}_{s}\right)-\int_{s}^{t \wedge \tau} L(g)\left(\bar{D}_{r}\right) d r
$$

is a local martingale (starting at s). A semimartingale solution to the martingale problem associated with a diffusor $L$ is called a diffusion process (or simply a diffusion) of diffusor $L$.

When not strictly necessary, we omit the stopping time $\tau$ from the definition of solution to a martingale problem. Furthermore, unless otherwise stated, we consider the solution to the martingale problem starting at 0 .

The diffusor $L$ is standard if, whenever $g \in C^{\infty}(N)$ depends only on $t$,

$$
L(g)(t)=\frac{d g}{d t}(t) .
$$

Remark 4.2 If $X$ is a continuous local martingale of bounded variation such that $X_{0}=0$, then, by martingale property, $X_{t}=0$ for every $t \in \mathbb{R}_{+}$(see, e.g. [18]).

The next result shows that our definition of standard diffusor is a natural requirement.

Proposition $4.3 A$ diffusor $L$ is standard if there exists a diffusion $D$ of diffusor $L$.

Proof. If $g \in C^{\infty}(N)$ depends only on $t$, considering $\bar{D}_{t}=\left(t, D_{t}\right)$, we have that

$$
D_{t}^{g}=g\left(\bar{D}_{t}\right)-g\left(\bar{D}_{0}\right)-\int_{0}^{t} L(g)\left(\bar{D}_{s}\right) d s=g(t)-g(0)-\int_{0}^{t} L(g)\left(\bar{D}_{s}\right) d s,
$$

is a local martingale.

Being $g(t)-g(0)$ and $\int L(g)\left(\bar{D}_{s}\right) d s$ bounded variation processes, $D^{g}$ is a bounded variation local martingale and, by Remark [4.2, $D_{t}^{g}=0$, which implies that $g(t)-g(0)=\int L(g)\left(\bar{D}_{s}\right) d s$. By differentiating both sides of the latter equality with respect to $t$, we get

$$
\frac{d g}{d t}(t)=L(g)\left(\bar{D}_{t}\right)
$$

which means that $L(g)=d g / d t$, i.e. $L$ is standard.

In the following we associate with each martingale problem a well-defined module of codiffusors and we prove that this module is actually completely equivalent to the martingale problem. We start with a preliminary lemma. 
Lemma 4.4 Let $L$ be a standard diffusor and $D$ be a diffusion of diffusor L. For any $\mu \in S\left(\tau^{*} N\right)$ we consider the codiffusor $\lambda=\mu-\langle\mu, L\rangle d^{2} t$. Then $\int\left\langle\lambda, d D_{t}\right\rangle$ is a local martingale.

Proof. If $\mu=d^{2} h$ with $h \in C^{\infty}(N)$ the lemma reduces to the definition of a diffusion of diffusor $L$.

If $\mu$ is a generic codiffusor, by Theorem 2.4 there exist $f_{i}, g_{i} \in C^{\infty}(N)$ such that

$$
\mu=\sum_{i} g_{i} d^{2} f_{i}
$$

If we consider $\lambda_{i}=d^{2} f_{i}-L\left(f_{i}\right) d^{2} t$, we have that $\int\left\langle\lambda_{i}, d D_{t}\right\rangle$ is a local martingale and, being

$$
\lambda=\mu-\langle\mu, L\rangle d^{2} t=\sum_{i} g_{i} \lambda_{i}
$$

we find

$$
\int\left\langle\lambda, d D_{t}\right\rangle=\sum_{i} \int\left\langle g_{i} \lambda_{i}, d D_{t}\right\rangle=\sum_{i} \int g_{i}\left(\bar{D}_{t}\right) d\left(\int\left\langle\lambda_{i}, d D_{s}\right\rangle\right)_{t} .
$$

The latter integral is a local martingale, being a sum of Itô integrals along the real local martingales $\int\left\langle\lambda_{i}, d D_{s}\right\rangle$.

We recall that $\Lambda_{L} \subset S\left(\tau^{*} N\right)$ denotes the annihilator of the one-dimensional module $\mathfrak{L}_{L}$ generated by the diffusor $L \in S(\tau N)$.

Theorem 4.5 The semimartingale $D$ on $M$ is a diffusion of standard diffusor $L$, if and only if, for every $\lambda \in \Lambda_{L}$,

$$
\int\left\langle\lambda, d D_{t}\right\rangle
$$

is a local martingale.

Proof. By Lemma 4.4, if $D$ is a diffusion of standard diffusor $L$ and $\lambda \in \Lambda_{L}$, then $\int\left\langle\lambda, d D_{t}\right\rangle$ is a local martingale. Indeed we know that $\lambda-\langle\lambda, L\rangle d^{2} t$ integrated along $D$ is a local martingale and that $\langle\lambda, L\rangle=0$ because $\lambda \in \Lambda_{L}$.

Conversely, suppose that the semimartingale $D$ is such that, $\forall \lambda \in \Lambda_{L}, \int\left\langle\lambda, d D_{t}\right\rangle$ is a local martingale. Given $g \in C^{\infty}(N)$, we have $\lambda=d^{2} g-L(g) d^{2} t \in \Lambda_{L}$, being $\left\langle d^{2} t, L\right\rangle=1$ and $\left\langle d^{2} g, L\right\rangle=L(g)$. Hence

$$
\int\left\langle\left(d^{2} g-L(g) d^{2} t\right), d D_{t}\right\rangle=g(\bar{D})-g\left(\bar{D}_{0}\right)-\int L(g)\left(\bar{D}_{t}\right) d t
$$

is a local martingale. Since $g$ is a generic function in $C^{\infty}(N)$, then $D$ is a diffusion of diffusor $L$.

In Lemma 4.4 and Theorem 4.5 we have implicitly assumed that the stopping time $\tau$ of the diffusion $D$ is equal to $+\infty$. The general case can be recovered by using Proposition 2.10. In order to prove a sort of converse of Theorem 4.5. since we do not need the uniqueness of the solution to the martingale problem, instead of the well-posedness notion we introduce the following definition.

Definition 4.6 A diffusor $L$ is a good diffusor if, for any $t_{0} \in \mathbb{R}$ and $x_{0} \in M$, there exists at least one diffusion $D$, starting at $t_{0}$ and such that $D_{t_{0}}=x_{0}$ almost surely, solution to the martingale problem associated with $L$. 
Proposition 4.7 If $L$ is a good diffusor, then

$$
\Lambda^{\prime}:=\left\{\lambda \in S\left(\tau^{*} N\right) \mid \int\left\langle\lambda, d D_{t}\right\rangle \text { is a local martingale }\right\} \subseteq \Lambda_{L}
$$

Proof. Given $\lambda \in \Lambda^{\prime}$, by Lemma 4.4 $\lambda-\langle\lambda, L\rangle d^{2} t \in \Lambda^{\prime}$ and, being $\Lambda^{\prime}$ closed with respect to the sum, we have

$$
\langle\lambda, L\rangle d^{2} t=\lambda-\left(\lambda-\langle\lambda, L\rangle d^{2} t\right) \in \Lambda^{\prime} .
$$

Let $D^{x_{0}, t_{0}}$ be a diffusion starting at $t_{0}$ such that $D_{t_{0}}^{x_{0}, t_{0}}=x_{0}$. The integral

$$
\int\left\langle\left(\langle\lambda, L\rangle d^{2} t\right), d D_{t}^{x_{0}, t_{0}}\right\rangle=\int(\langle\lambda, L\rangle)\left(\bar{D}_{t}^{x_{0}, t_{0}}\right) d t
$$

is a bounded variation process and also a local martingale and, by Remark 4.2 ,

$$
\int_{t_{0}}^{r}(\langle\lambda, L\rangle)\left(\bar{D}_{t}^{x_{0}, t_{0}}\right) d t=0
$$

for any $r>t_{0}$. Since $(\langle\lambda, L\rangle)\left(\bar{D}_{t}^{x_{0}, t_{0}}\right)$ is continuous with respect to $t$, we have that $(\langle\lambda, L\rangle)\left(\bar{D}_{t}^{x_{0}, t_{0}}\right)=$ 0 and, considering the limit for $t \rightarrow t_{0}$ in the previous expression, we get $(\langle\lambda, L\rangle)\left(t_{0}, x_{0}\right)=0$. Since $x_{0} \in M$ and $t_{0} \in \mathbb{R}$ are generic points the proposition is proved.

Corollary 4.8 If $L$ is a good diffusor, then

$$
\Lambda_{L}=\left\{\lambda \in S\left(\tau^{*} N\right) \mid \int\left\langle\lambda, d D_{t}\right\rangle \text { is a local martingale }\right\} .
$$

In the following we always consider good diffusors $L$. This choice is not restrictive since, using the stopping time $\tau$ and our definition of solution to the martingale problem, we can exploit all existence results for diffusion processes in $\mathbb{R}^{n}$ (see [22]).

\section{Symmetries of diffusions}

Generalizing the natural idea of symmetries of ODEs as diffeomorphisms transforming solutions into solutions, we give the following definition.

Definition 5.1 Let $\bar{\Phi}: N \rightarrow N$ be an invertible semimartingale transformation. The diffeomorphism $\bar{\Phi}=(f, \Phi)$ is a symmetry of the diffusions associated with $L$ (in short, a symmetry of $L$ ) if, for any diffusion $D$ of diffusor $L$, also $\Phi_{f}(D)$ is a diffusion of diffusor $L$.

The next result characterizes symmetries of diffusions associated with a diffusor $L$ in terms of a suitable invariance property of the module of codiffusors $\Lambda_{L}$.

Theorem 5.2 An invertible semimartingale transformation $\bar{\Phi}: N \rightarrow N$ is a symmetry of $L$ if and only if $\bar{\Phi}$ is a symmetry of $\Lambda_{L}$.

Proof. Suppose that $\bar{\Phi}$ is a symmetry of $L$, and let $D$ be any diffusion of diffusor $L$. Obviously, by Theorem 4.5 and by the definition of symmetry, $\forall \lambda \in \Lambda_{L}$

$$
\int\left\langle\lambda, d \Phi_{f}(D)_{t}\right\rangle
$$


is a local martingale. On the other hand, by Theorem 3.14 we have

$$
\int\left\langle\lambda, d \Phi_{f}(D)_{t}\right\rangle=H_{f}\left(\int\left\langle\bar{\Phi}^{*}(\lambda), d D_{t}\right\rangle\right) .
$$

Since the latter equality and Proposition 4.7 ensure that $\bar{\Phi}^{*}(\lambda) \in \Lambda_{L}$, then $\bar{\Phi}^{*}\left(\Lambda_{L}\right) \subseteq \Lambda_{L}$. The equality follows from the invertibility of $\bar{\Phi}$.

Conversely, suppose that $\bar{\Phi}^{*}\left(\Lambda_{L}\right)=\Lambda_{L}$ and let $D$ be any diffusion of diffusor $L$. Fixing $\lambda \in \Lambda_{L}$, from Theorem 3.14 we have

$$
\int\left\langle\lambda, d \Phi_{f}(D)_{t}\right\rangle=H_{f}\left(\int\left\langle\bar{\Phi}^{*}(\lambda), d D_{t}\right\rangle\right) .
$$

Since $\bar{\Phi}^{*}(\lambda) \in \Lambda_{L}$, the right-hand side of the last equality is a local martingale. Then, by Theorem 4.5. $\Phi_{f}(D)$ is a diffusion of diffusor $L$.

In order to provide a simpler characterization of symmetries of $L$ we give the following lemma.

Lemma 5.3 Let $L$ be a standard diffusor. If there exists a diffusor $L^{\prime}$ such that, $\forall \lambda \in \Lambda_{L}$, $\left\langle\lambda, L^{\prime}\right\rangle=0$, then there exists $\mu \in C^{\infty}(N)$ such that $L^{\prime}=\mu L$.

Proof. Let us consider $\widetilde{L}=L^{\prime}-L^{\prime}(t) L$ : we show that $\widetilde{L}=0$ proving that, $\forall g \in C^{\infty}(N)$, $\widetilde{L}(g)=0$. Since $L$ is standard, $L(t)=\left\langle d^{2} t, L\right\rangle=1$ and

$$
\begin{aligned}
\widetilde{L}(t) & =\left\langle d^{2} t, L^{\prime}\right\rangle-L^{\prime}(t)\left\langle d^{2} t, L\right\rangle \\
& =L^{\prime}(t)-L^{\prime}(t) L(t)=0 .
\end{aligned}
$$

Obviously, if $\lambda \in \Lambda_{L}$, then $\langle\lambda, \widetilde{L}\rangle=0$. So, if $g \in C^{\infty}(M)$, then $\lambda=d^{2} g-L(g) d^{2} t \in \Lambda_{L}$. Therefore

$$
\begin{aligned}
\widetilde{L}(g) & =\left\langle\left(\lambda+L(g) d^{2} t\right), \widetilde{L}\right\rangle \\
& =\langle\lambda, \widetilde{L}\rangle+L(g) \widetilde{L}(t)=0,
\end{aligned}
$$

and, by Theorem 2.6] the last statement is equivalent to $\widetilde{L}=0$.

With the notations and the hypothesis of Theorem $5.2, \bar{\Phi}$ is a symmetry of $L$ if and only if $\bar{\Phi}^{*}(L)=\mu L$, for some $\mu \in C^{\infty}(M)$ such that $\mu \neq 0$.

Definition 5.4 Let $X$ be a complete vector field on $N$ with corresponding flow $\bar{\Phi}_{a}$. The vector field $X$ is an infinitesimal symmetry for the diffusions associated with a diffusor $L$ (in short an infinitesimal symmetry for $L$ ) if, $\forall a \in \mathbb{R}, \bar{\Phi}_{a}$ is a symmetry of the diffusor $L$.

Remark 5.5 A necessary condition for $X$ to be an infinitesimal symmetry of a diffusor $L$ is that the flow $\bar{\Phi}_{a}$ is a one-parameter group of invertible semimartingale transformations. This is equivalent to require that $X$ is projectable, i.e. the vector field $X$ is of the form $X=\phi^{i} \partial_{x^{i}}+\tau \partial_{t}$, where the function $\tau$ depends only on $t$.

Theorem 5.6 A projectable complete vector field $X$ is an infinitesimal symmetry of a standard diffusor $L$ if and only if $X$ is a symmetry of $\Lambda_{L}$, i.e.

$$
\mathcal{L}_{X}\left(\Lambda_{L}\right) \subseteq \Lambda_{L}
$$

Proof. The necessity (and the sufficiency) of the existence of the flow and of the projectability of $X$ are explained in Remark 5.5

Besides, since $\Lambda_{L}$ is a $k$-dimensional module (with $k=\operatorname{rank}\left(\tau^{*} N\right)-1$ ) the necessity and sufficiency of condition (10) are simple consequences of Theorem 3.10 and Theorem 5.2 .

The following proposition provides a very useful condition, ensuring that a complete vector field is a symmetry of a diffusion $L$. 
Proposition 5.7 Let $X$ be a projectable complete vector field and $L$ be a standard diffusor. Then $\mathcal{L}_{X}\left(\Lambda_{L}\right) \subseteq \Lambda_{L}$ if and only if there exists $\mu \in C^{\infty}(N)$ such that

$$
\mathcal{L}_{X}(L)=\mu L
$$

Proof. Suppose that $\mathcal{L}_{X}\left(\Lambda_{L}\right) \subseteq \Lambda_{L}$. For any codiffusor $\lambda \in \Lambda_{L}$, we have

$$
\begin{aligned}
0 & =\mathcal{L}_{X}(\langle\lambda, L\rangle) \\
& =\left\langle\mathcal{L}_{X}(\lambda), L\right\rangle+\left\langle\lambda, \mathcal{L}_{X}(L)\right\rangle \\
& =\left\langle\lambda, \mathcal{L}_{X}(L)\right\rangle
\end{aligned}
$$

Hence, by Lemma 5.3. there exists $\mu \in C^{\infty}(N)$ such that $\mathcal{L}_{X}(L)=\mu L$. Conversely, suppose that $\mathcal{L}_{X}(L)=\mu L$; then for any $\lambda \in \Lambda_{L}$,

$$
\begin{aligned}
0 & =\left\langle\mathcal{L}_{X}(\lambda), L\right\rangle+\langle\lambda, \mu L\rangle \\
& =\left\langle\mathcal{L}_{X}(\lambda), L\right\rangle
\end{aligned}
$$

Hence $\mathcal{L}_{X}(\lambda) \in \Lambda_{L}$, completing the proof.

In order to give a coordinate expression for condition (11) we consider a coordinate system $x^{i}$ on $M$ and a standard diffusor $L$ of the form

$$
L=A^{i j} \partial_{x^{i} x^{j}}+b^{i} \partial_{x^{i}}+A^{i t} \partial_{x^{i} t}+\partial_{t} .
$$

It is easy to prove that, if $L$ is a good diffusor, then $A^{i t}=0$ and the matrix $A^{i j}$ is semidefinite positive. Hence $L$ has the form

$$
L=A^{i j} \partial_{x^{i} x^{j}}+b^{i} \partial_{x^{i}}+\partial_{t} .
$$

Given a projectable vector field $X=\phi^{i} \partial_{x^{i}}+\tau \partial_{t}$, we can calculate $\mathcal{L}_{X}(L)$ and, inserting this expression in (11), we obtain $\mu=-\partial_{t} \tau$ and

$$
\begin{gathered}
\phi^{k} \partial_{x^{k}} A^{i j}+\tau \partial_{t} A^{i j}-A^{i k} \partial_{x^{k}} \phi^{j}-A^{k j} \partial_{x^{k}} \phi^{i}+A^{i j} \partial_{t} \tau=0 \\
\phi^{k} \partial_{x^{k}} b^{i}+\tau \partial_{t} b^{i}-b^{k} \partial_{x^{k}} \phi^{i}-A^{j k} \partial_{x^{j} x^{k}} \phi^{i}+b^{i} \partial_{t} \tau-\partial_{t} \phi^{i}=0,
\end{gathered}
$$

for $i, j=1, \ldots, \operatorname{dim}(M)$.

In the following we compare the symmetry approach proposed in this paper, and in particular the determining equations (13) and (14), with other results on symmetries of stochastic processes appearing in the literature.

Given a diffusor $L$, it is natural to consider the corresponding Kolmogorov equation

$$
L(u)=A^{i j} \partial_{x^{i} x^{j}}(u)+b^{i} \partial_{x^{i}}(u)+\partial_{t}(u)=0
$$

describing the behaviour of the mean value of regular functions of the solution process $X_{t}$. More precisely, a solution $u(x, t)$ to equation (15) is of the form $\mathbb{E}\left[f\left(X_{T}\right) \mid X_{t}=x\right]=u(x, t)$, with $t \in[0, T]$. Since (15) is a PDE, its Lie symmetries can be interpreted as vector fields on $J^{0}(N, \mathbb{R})$ of the form

$$
Z=\tau(x, t, u) \partial_{t}+\phi^{i}(x, t, u) \partial_{x^{i}}+\psi(x, t, u) \partial_{u}
$$


satisfying (in the non-degenerate case, i.e. when $A^{i j}$ has maximal rank) the following conditions (see e.g. [8, 17])

$$
\begin{gathered}
\psi(x, t, u)=h(x, t) u \\
\partial_{u}\left(\phi^{i}\right)=0 \\
\partial_{u}(\tau)=0 \\
\partial_{x^{i}}(\tau)=0 \\
\partial_{t}(h)+A^{i j} \partial_{x^{i} x^{j}}(h)+b^{i} \partial_{x^{i}}(h)=0 \\
\phi^{k} \partial_{x^{k}} A^{i j}+\tau \partial_{t} A^{i j}-A^{i k} \partial_{x^{k}} \phi^{j}-A^{k j} \partial_{x^{k}} \phi^{i}+A^{i j} \partial_{t} \tau=0 \\
\phi^{k} \partial_{x^{k}} b^{i}+\tau \partial_{t} b^{i}-b^{k} \partial_{x^{k}} \phi^{i}-A^{j k} \partial_{x^{j}} x^{k} \phi^{i}+b^{i} \partial_{t} \tau-\partial_{t} \phi^{i}+A^{i k} \partial_{x^{k}}(h)+A^{k i} \partial_{x^{k}}(h)=0 .
\end{gathered}
$$

It is interesting to note that these equations coincide with equations (13) and (14) when $h$ is constant. This is due to the fact that, in our approach, the main object is the process $X_{t}$ and a symmetry $Y$ on $M$ transforms the solution $X_{t}$ to the martingale problem into a (possibly different) solution $\Phi_{f}(X)_{t}$ to the same martingale problem. Hence $Y=Z$ (under the hypothesis $h=0$ ) transforms solutions to (15) into other solutions to (15).

Indeed a solution $u$ to the Kolmogorov equation such that $u(x, T)=g(x)$ is of the form $u(x, t)=$ $\mathbb{E}\left[g\left(X_{T}\right) \mid X_{t}=x\right]$. This means that

$$
\begin{aligned}
u\left(\Phi_{a}^{-1}(x, t), f_{a}^{-1}(t)\right) & =\mathbb{E}\left[g\left(X_{T}\right) \mid X_{f_{a}^{-1}(t)}=\Phi_{a}^{-1}(x)\right] \\
& =\mathbb{E}\left[g\left(X_{T}\right) \mid \Phi_{a}\left(H_{f_{a}}(\bar{X})\right)=x\right] \\
& =\mathbb{E}\left[g \circ \Phi_{a}^{-1}\left(\Phi_{a, f_{a}}(X)_{f_{a}^{-1}(T)}, f_{a}^{-1}(T)\right) \mid \Phi_{a, f_{a}}(X)_{t}=x\right]=v(x, t) .
\end{aligned}
$$

Since $\Phi_{f}(X)$ is still a solution to the same martingale problem, we have that $v$ is the unique solution to $L(v)=0$ with final condition $v\left(x, f_{a}^{-1}(T)\right)=g\left(\Phi_{a}^{-1}\left(x, f_{a}^{-1}(T)\right)\right)$.

The fact that only the transformations with $h=0$ turn out to be symmetries of both the diffusion process and the Kolmogorov equation follows from the fact that the transformations of the function $u$ do not have a natural meaning when the focus is on the process.

Another natural comparison arising in this framework is the study of the relationship between the symmetries of a martingale problem as proposed in the present paper and the symmetries of the corresponding SDE as given in 3]. Since in [3] we consider only autonomous SDEs and stochastic time changes, in order to make the two approaches correctly comparable, we restrict our considerations to autonomous diffusions (i.e. $A^{i j}, b^{i}$ not depending on $t$ ) and time changes of the form $\tau=a t$ for some $a \in \mathbb{R}$.

Given $\mu: M \rightarrow \mathbb{R}^{n}$ and $\sigma: M \rightarrow \operatorname{Mat}(n, m)$, in [3] we consider SDEs of the form

$$
d X_{t}=\mu^{i}\left(X_{t}\right) d t+\sigma_{\alpha}^{i}\left(X_{t}\right) d W^{\alpha}
$$

where $(\mu, \sigma)=\left(\mu^{i}(x), \sigma_{\alpha}^{i}(x)\right)$, and $m$ is the dimension of the Brownian motion driving the SDE. The relationship between $(\mu, \sigma)$ and $\left(b^{i}, A^{i j}\right)$ is provided by Itô formula which ensures that

$$
b^{i}=\mu^{i}, \quad A^{i j}=\frac{1}{2} \sum_{\alpha=1}^{m} \sigma_{\alpha}^{i} \sigma_{\alpha}^{j} .
$$

The infinitesimal stochastic transformation of a process $X$ and of a Brownian motion $W$ is given by a triple $(\tilde{Y}, C, a)$ where $\tilde{Y}=\phi^{i} \partial_{x^{i}}$ is a vector field on $M$, describing the spatial change of $X$, $C: M \rightarrow \mathfrak{s o}(m)$ is a function representing the random rotation of the Brownian motion $W$ and 
taking values in the group of antisymmetric matrices, and $a \in \mathbb{R}$ is the parameter of the time rescaling.

The determining equations for $(\tilde{Y}, C, a)$ are

$$
\begin{gathered}
\phi^{k} \partial_{x^{k}} \mu^{i}-\mu^{k} \partial_{x^{k}} \phi^{i}-\frac{1}{2} \sum_{\alpha} \sigma_{\alpha}^{j} \sigma_{\alpha}^{k} \partial_{x^{j} x^{k}} \phi^{i}+a \mu^{i}=0, \\
\phi^{k} \partial_{x^{k}}\left(\sigma_{\alpha}^{i}\right)-\sigma_{\alpha}^{k} \partial_{x^{k}}\left(\phi^{i}\right)+C_{\alpha}^{\beta} \sigma_{\alpha}^{i}+\frac{1}{2} a \sigma_{\alpha}^{i}=0 .
\end{gathered}
$$

It is easy to check, by using (16), that equation (17) coincides with equation (14) with $Y=\tilde{Y}+a t \partial_{t}$ and that, being $C$ antisymmetric, equation (18) implies equation (12). Furthermore, it is possible to prove that, if $A^{i j}$ has constant rank, there exists a unique antisymmetric matrix $C(x)$ such that, if $Y=\tilde{Y}+a t \partial_{t}$ solves equation (13), then $(\tilde{Y}, C, a)$ solves equation (18). Therefore, providing $A$ is non-degenerate, the symmetries of a SDE with deterministic time change defined in [3] are in one-to-one correspondence with the symmetries of the related martingale problem introduced here. The presence of the matrix $C \neq 0$ is essential for the validity of this correspondence. Indeed, since in the martingale problem formulation the Brownian motion is not fixed, freezing the Brownian motion in the SDE formulation by choosing $C=0$ may cause the loss of some Lie symmetries (see [7] and [3] for further details).

\section{Acknowledgements}

The authors would like to thank Prof. Gaeta for his useful comments and suggestions in the first part of the work. This work was supported by Gruppo Nazionale Fisica Matematica (GNFMINdAM).

\section{References}

[1] Michel Cohen de Lara. Geometric and symmetry properties of a nondegenerate diffusion process. Ann. Probab., 23(4):1557-1604, 1995.

[2] Francesco C. De Vecchi, Paola Morando, and Stefania Ugolini. Reduction and reconstruction of stochastic differential equations via symmetries. J. Math. Phys., 57(12):123508, 22, 2016.

[3] Francesco C. De Vecchi, Paola Morando, and Stefania Ugolini. Symmetries of stochastic differential equations: a geometric approach. J. Math. Phys., 57(6):063504, 17, 2016.

[4] K. David Elworthy. Stochastic differential equations on manifolds, volume 70 of London Mathematical Society Lecture Note Series. Cambridge University Press, Cambridge-New York, 1982.

[5] Michel Émery. Stochastic calculus in manifolds: With an appendix by P.-A. Meyer. Universitext. Springer-Verlag, Berlin, 1989.

[6] Ebrahim Fredericks and Fazal Mahmood Mahomed. Symmetries of first-order stochastic ordinary differential equations revisited. Math. Methods Appl. Sci., 30(16):2013-2025, 2007.

[7] Giuseppe Gaeta. Lie-point symmetries and stochastic differential equations. II. J. Phys. A, 33(27):4883-4902, 2000.

[8] Giuseppe Gaeta and Niurka Rodríguez Quintero. Lie-point symmetries and stochastic differential equations. J. Phys. A, 32(48):8485-8505, 1999. 
[9] Joseph Glover and Joanna Mitro. Symmetries and functions of Markov processes. Ann. Probab., 18(2):655-668, 1990.

[10] Elton P. Hsu. Stochastic analysis on manifolds, volume 38 of Graduate Studies in Mathematics. American Mathematical Society, Providence, RI, 2002.

[11] Nobuyuki Ikeda and Shinzo Watanabe. Stochastic differential equations and diffusion processes, volume 24 of North-Holland Mathematical Library. North-Holland Publishing Co., Amsterdam-New York; Kodansha, Ltd., Tokyo, 1981.

[12] Roman Kozlov. Symmetries of systems of stochastic differential equations with diffusion matrices of full rank. J. Phys. A, 43(24):245201, 16, 2010.

[13] Joan-Andreu Lázaro-Camí and Juan-Pablo Ortega. Reduction, reconstruction, and skewproduct decomposition of symmetric stochastic differential equations. Stoch. Dyn., 9(1):1-46, 2009.

[14] Paul Lescot and Jean-Claude Zambrini. Isovectors for the Hamilton-Jacobi-Bellman equation, formal stochastic differentials and first integrals in Euclidean quantum mechanics. In Seminar on Stochastic Analysis, Random Fields and Applications IV, volume 58 of Progr. Probab., pages 187-202. Birkhäuser, Basel, 2004.

[15] Ming Liao. Symmetry groups of Markov processes. Ann. Probab., 20(2):563-578, 1992.

[16] Paul-André Meyer. Géométrie stochastique sans larmes. In Seminar on Probability, XV (Univ. Strasbourg, Strasbourg, 1979/1980) (French), volume 850 of Lecture Notes in Math., pages 44-102. Springer, Berlin-New York, 1981.

[17] Peter J. Olver. Applications of Lie groups to differential equations, volume 107 of Graduate Texts in Mathematics. Springer-Verlag, New York, second edition, 1993.

[18] L. Chris G. Rogers and David Williams. Diffusions, Markov processes, and martingales. Vol. 2. Wiley Series in Probability and Mathematical Statistics: Probability and Mathematical Statistics. John Wiley \& Sons, Inc., New York, 1987. Itô calculus.

[19] Laurent Schwartz. Géométrie différentielle du 2ème ordre, semi-martingales et équations différentielles stochastiques sur une variété différentielle. In Seminar on Probability, XVI, Supplement, volume 921 of Lecture Notes in Math., pages 1-148. Springer, Berlin-New York, 1982.

[20] Boonlert Srihirun, Sergey V. Meleshko, and Eckart Schulz. On the definition of an admitted Lie group for stochastic differential equations. Commun. Nonlinear Sci. Numer. Simul., 12(8):1379-1389, 2007.

[21] Hans Stephani. Differential equations: Their solution using symmetries. Cambridge University Press, Cambridge, 1989.

[22] Daniel W. Stroock and S. R. Srinivasa Varadhan. Multidimensional diffusion processes, volume 233 of Grundlehren der Mathematischen Wissenschaften [Fundamental Principles of Mathematical Sciences]. Springer-Verlag, Berlin-New York, 1979.

[23] Gazanfer Ünal and Jian-Qiao Sun. Symmetries and conserved quantities of stochastic dynamical control systems. Nonlinear Dynam., 36(1):107-122, 2004. 